\title{
Business Process Management y Seis Sigma en el análisis de procesos: caso de estudio
}

\author{
Rincon García, Nicolas* \\ Aguirre Mayorga, Hugo Santiago** \\ Caballero Villalobos, Juan Pablo***
}

\section{Resumen}

En el análisis y mejoramiento de procesos pueden emplearse diferentes aproximaciones donde Business Process Management y Seis Sigma se encuentran entre las principales metodologías empleadas por las organizaciones. Si bien estas metodologías tienen enfoques diferentes, no son excluyentes y en la literatura se recomienda su uso complementario, dado que Business Process Management tiene un enfoque cualitativo y un componente de integración con los sistemas de información que soportan los procesos, mientras que las herramientas cuantitativas de Seis Sigma se emplean para valorar las oportunidades de mejora. En la presente investigación de desarrolla un caso de estudio en un proceso de compras de una organización educativa con el objetivo de analizar cómo se pueden complementar estas metodologías mediante el análisis de manuales de trabajo, entrevistas, análisis del soporte informático y análisis estadístico. El análisis cualitativo de Business Process Management permite alinear el proyecto de mejoramiento con la estrategia organizacional y sus herramientas de análisis del flujo de proceso y sistemas de información permiten identificar la información necesaria para ser analizada con herramientas cuantitativas de Seis Sigma, las cuales permite establecer las variables críticas que deben controlarse para mejorar el proceso y estimar las posibles mejoras. En el caso de estudio se comprueba que las dos metodologías pueden complementarse.

Palabras clave: Business Process Management, seis sigma, análisis de procesos.

\section{Recibido: 15-09-12 Aceptado: 10-05-14}

* Profesor Instructor de la Pontificia Universidad Javeriana, Departamento de Ingeniería Industrial. M.Sc. Logistics and Supply Chain Management, University of Westminster, Estudiante doctoral, School of Civil Engineering and the Environment, University of Southampton. E-mail: nicolas.rincon@javeriana.edu.co, Autor para la correspondecia.

** Profesor Asociado de la Pontificia Universidad Javeriana, Departamento de Ingeniería Industrial. M.Sc. Universidad de los Andes, candidato a PhD en Ingeniería, Pontíficia Universidad Javeriana. E-mail:saguirre@javeriana.edu.co

*** Profesor Asociado de la Pontificia Universidad Javeriana, Departamento de Ingeniería Industrial. M.Sc. Universidad de los Andes, candidato a PhD en Ingeniería, Universidad de los Andes. E-mail:juan.caballero@javeriana.edu.co 


\section{Business Process Management and Six Sigma in Process Analysis: A Case Study}

\section{Abstract}

In the analysis and improvement of processes, different approaches can be used; Business Process Management and Six Sigma are found among the principle methodologies employed by organizations. Although these methodologies have different approaches, there are not mutually exclusive. In the literature, their complementary used is recommended, given that Business Process Management has a qualitative approach and a component of integration with the information systems that support the processes, while the quantitative tools of Six Sigma are used to evaluate the opportunities for improvement. This research develops a case study in the purchase process for an educational organization with the objective of analyzing how these methodologies can complement each other through the analysis of work manuals, interviews, analysis of informatics support and statistical analysis. The qualitative analysis of Business Process Management permits aligning the project for improvement with the organizational strategy and its analysis tools for process flow and information systems make it possible to identify the information needed to be analyzed with the quantitative tools of Six Sigma, which permits establishing the critical variables that ought to be controlled to improve the process and estimate the possible improvements. In this case study, it is proven that the two methodologies complement each other.

Keywords: Business Process Management, Six Sigma, process analysis.

\section{Introducción}

Business Process Management (BPM) ha venido empleándose desde finales de la década del 90 para la mejora de los procesos (Jeston y Nelis, 2006), contando con un creciente interés por parte de empresas y desarrolladores de software (Ko et al., 2009). De acuerdo con el último estudio de Process Excellence Network (2013) BPM se encuentra dentro de las tres principales metodologías de mayor uso por parte de las organizaciones en los programas de "Excelencia en Procesos" junto con Lean y Lean Seis Sigma.

La interpretación de BPM puede variar desde un sistema orientado a la tecnología para el apoyo de procesos operacionales basado en la evolución de herramientas de workflow (van der Aalst et al., 2003a; Ko et al., 2009) hasta una disciplina gerencial que integra tecnologías de información y procesos de negocio con el fin de crear ventaja competitiva mediante la visión transversal, medición y alineación estratégica (Lederer et al., 2011; Harmon, 2007).

BPM cuenta con una serie de estándares para su implementación. Ko et al., (2009), los clasifica en 4 categorías: gráfico, ejecución, intercambio y diagnóstico. Los tres primeros han sido implementados en Suites de BPM (BPMS), software que puede tener elementos para la modelación, integración, automatización, administración, análisis y simulación (Mooney, 2006), mientras que con respecto al estándar de diagnóstico, hace faltan ciertos desarrollos dado que la metodología no 
cuenta con herramientas cuantitativas per se. La falta de métricas en proyectos de BPM es una preocupación mayor en las empresas, especialmente en empresas de servicios, pues se corre el riesgo de no obtener impactos significativos frente a los costos de realizar el proyecto de mejora (Neubauer, 2009).

Diferentes autores (van der Aalst et al., 2003a; Ko et al., 2009; Lederer et al., 2011; Harmon, 2007) coinciden en que la fase de diagnóstico es un componente básico para la mejora de los procesos. Dado que BPM no cuenta con un estándar para el diagnóstico (Ko et al., 2009), se emplean herramientas de otras metodologías para entender el desempeño de los procesos e identificar y cuantificar las oportunidades de mejora. Dentro de estas metodologías se encuentran Seis Sigma, Lean (Neubauer, 2009) y Minería de Procesos (Aguirre et al., 2013).

Seis Sigma basa la mejora de procesos en métodos estadísticos y en el enfoque científico para reducir el índice de defectos declarados por el cliente, identificando de manera cuantitativa las causas principales que generan las no conformidades (Linderman et al., 2003). Seis Sigma ha sido una metodología ampliamente utilizada en empresas de manufactura y actualmente en empresas de servicios, empleando herramientas estadísticas para el diagnóstico, análisis y mejora de los procesos (Aboelmaged, 2010).

Harmon, (2007), Neubauer (2009) y Johansen et al., (2010) proponen el uso de Seis Sigma como herramienta cuantitativa para soportar el enfoque cualitativo de BPM, sin embargo en la revisión bibliográfica realizada por los autores de esta investigación no se encontraron casos de estudio que muestren en detalle cómo integrar estas metodologías en empresas de servicios que empleen sistemas de información integrados como soporte de sus procesos.

En este trabajo se enfatiza el caso de estudio como método de investigación que gracias a su capacidad única de tratar con diferentes tipos de evidencias: documentos, entrevistas, observaciones, con el fin de mostrar posibles resultados dada una situación inicial y una serie de eventos y decisiones (Yin, 2003). Los casos de estudio son ampliamente utilizados en las ciencias administrativas para probar teorías en instancias concretas y brindar experiencias enriquecedoras a profesionales e investigadores (Dubois y Araujo, 2007).

En esta investigación, se asume como unidad de estudio el proceso de compras en una institución universitaria, el cual está soportado en un sistema ERP (Enterprise Resource Planning). Este es un proceso crítico para la organización dado que tiene un alto impacto en el indicador de satisfacción de los empleados al darles los recursos para cumplir con su labor. Por otro lado se debe considerar que es un proceso complejo por ser de servicio y con alta interacción humana (Johannsen et al., 2010). Según Stanley y Wisner (2002), con la mejora de la calidad del proceso de compras se pueden generar beneficios duraderos como incremento de la rentabilidad y ahorros.

Se pretende responder indagar sobre la forma de integrar la metodología de rediseño de procesos de BPM con técnicas de Seis Sigma para diagnosticar cualitativamente y cuantitativamente un proceso de negocio que esté soportado en 
un sistema de información integrado. Para ello, se revisó la literatura sobre las tendencias en BPM y el uso de técnicas Seis Sigma en empresas de servicios.

Como proposiciones se plantea: 1 ) la metodología BPM permite entender y documentar tanto los procesos como los proyectos de mejora, de forma que estén alineados con la estrategia de la compañía. El uso de herramientas gráficas y el análisis del soporte informático permite identificar la información relevante para el análisis de los indicadores de gestión, y 2) las herramientas estadísticas de la metodología Seis Sigma permiten cuantificar el estado inicial de los procesos y validar las hipótesis de mejora obtenidas.

La metodología propuesta para desarrollar el caso de estudio se basa en la mencionada por Harmon (2007) para el mejoramiento de procesos. Se parte de la revisión de la literatura en BPM, Seis Sigma y trabajos relacionados, así como de la documentación de procesos de la organización donde se encuentran la estrategia, flujos de proceso, responsables e indicadores previamente establecidos. Se realizan entrevistas no estructuradas a los diferentes responsables del proceso de compras para validar la documentación e identificar las posibles oportunidades de mejora.

Con la información recopilada, se realiza un análisis del flujo de proceso y del sistema de información para establecer métricas e identificar información relevante. Se explican las características del caso de estudio y se presentan los análisis cuantitativo y cualitativo para el diagnóstico y análisis de procesos donde se combinan las técnicas de BPM y Seis Sigma.
Se emplea la información del sistema para realizar el análisis estadístico con la herramienta Seis Sigma. La validación del caso de estudio se confirma al poder encontrar un soporte estadístico con herramientas Seis Sigma a las posibles oportunidades de mejora identificadas de forma cualitativa con herramientas de BPM.

\section{Business Process Management}

Dentro de los casos de estudio que emplean la metodología BPM se encuentran diferentes enfoques dada la visión de BPM y el nivel de madurez de los procesos. Al respecto, Fernando-Sentanin et al., (2008) muestra como en un centro de investigación en Brasil se emplea BPM como herramienta para analizar y documentar la estrategia de la organización con el fin de estandarizar los procesos en la organización.

El uso de BPM relacionado con la visión de automatización de procesos se encuentra en Küng y Hagen (2007); Seetharaju y Marjanovic (2009); Hesson et al., (2007); Climent et al., (2009), quienes ubican como elemento común el análisis de flujo del proceso en aras de la eliminación o reducción de tareas que no agregan valor, indicando los resultados una disminución del tiempo de ciclo del proceso.

Para Rebuge y Ferreira (2012) es necesario el empleo de la minería de datos para el análisis de procesos automatizados en organizaciones del sector salud. Los autores encontraron en sus investigaciones que las herramientas de diagnóstico deben ser seleccionadas y 
modificadas de acuerdo a las especificaciones del proceso con el fin de identificar patrones y cuellos de botella.

Por otro lado, Aguirre et al., (2013) muestra la aplicación de técnicas de minería de procesos y simulación para analizar las variables que influyen en los tiempos de ciclo de un proceso de compras.

La importancia de tener en cuenta los ejecutores del proceso es presentada por Seetharahu y Marjanovic (2009), quien encontró oportunidades de mejora al entender las actividades del proceso, las reglas de negocio y la visión de los ejecutores del proceso aprovechando su experiencia con el fin de automatizar el proceso de compras. Si bien se reconoce la importancia del análisis cualitativo y cuantitativo, en las investigaciones revisadas no se muestra el diagnóstico detallado del proceso, donde se analice el proceso de manera cualitativa y cuantitativa, documentando las diferentes etapas del diagnóstico e identificando oportunidades de mejora que surgen a partir del enfoque de BPM combinado con herramientas cuantitativas.

Según Ko et al., (2009) BPM está siendo reconocido como una práctica de mejora con un soporte tecnológico que brinda agilidad al rediseño de procesos. Bajo este criterio Harmon (2007) afirma que BPM es una filosofía donde las compañías organizadas confían en el concepto de proceso para entender el negocio y organizar la mejora continua. Los beneficios obtenidos son: mejora de la eficiencia y la efectividad, incremento de la agilidad, implementación de nueva tecnología y promoción de una nueva filosofía gerencial.

Dentro de los proyectos de BPM se encuentran una serie de métodos, técni- cas y software para diseñar, controlar, analizar, mejorar y documentar procesos operacionales teniendo en cuenta los factores humanos, organizacionales y plataformas tecnológicas (Neubauer, 2009). El software de BPM se denominan BPMS (Business Process Management Systems) y cuatro elementos en el ciclo de la metodología: diagnóstico, diseño de proceso, configuración del sistema y mejora del proceso, este último resultado de la automatización.

La etapa de diagnóstico se basa en el análisis del flujo del proceso y cuenta con tres elementos básicos (van der Aalst et al., 2003b): 1) Validación, consiste en el análisis entre el flujo del proceso deseado y real, pueden emplearse herramientas de simulación; 2) verificación, basada en el análisis de la lógica del flujo del proceso soportado en estructuras de grafos (van der Aalst et al.,2003b) y redes de Petri (Han et al., 2009) y; 3) análisis de desempeño, consistente en la identificación de oportunidades de mejora basándose en las métricas del proceso e identificación de cuellos de botella del sistema, donde pueden emplearse técnicas tradicionales de calidad.

El énfasis del análisis de flujo del proceso como diagnóstico en BPM ha migrado hacia una visión más integral de negocio, donde debe tenerse en cuenta el impacto del proyecto en el cumplimiento de la estrategia general de la compañía (Han et al., 2009).

La etapa de diagnóstico presenta varias fases interrelacionadas, inicia con la relacionada al estudio de la situación actual del proceso, luego la recolección de información, seguidamente la definición del proceso basado en las expectativas de los 
clientes donde son detectados los problemas cuyo fin radica en el establecimiento de las hipótesis que reflejan su causa raíz para planear las soluciones (Evans y Lindsay, 2000). Adicionalmente, se identifica el impacto del proyecto en el desempeño del proceso y los indicadores estratégicos de la compañía (Han et al., 2009; Jeston y Nelis, 2006; Harmon, 2007).

Dentro del diagnóstico, análisis y seguimiento de los procesos se encuentran herramientas de minería de procesos y de monitoreo de actividades de negocio o Business Activity Monitoring (BAM). Las técnicas de minería de procesos permiten descubrir el modelo de ejecución real de los procesos y patrones de comportamiento (Aguirre et al., 2013), no obstante estas técnicas requieren herramientas especializadas para su aplicación (Tiwari et al., 2008), las cuales han venido madurando con el pasar de los años.
El componente BAM de los sistemas BPMS permite aplicar la inteligencia operacional e integración de aplicaciones tecnológicas para alertar a los integrantes de la organización sobre cambios en el desempeño de los procesos que pueden requerir atención. Al analizar las actividades en tiempo real se pueden tomar mejores decisiones, identificar rápidamente problemas e implementar acciones antes de que se vea seriamente afectado el proceso (Jiang et al., 2007). Para ello es necesario configurar el sistema de alarmas de acuerdo con las necesidades específicas de los procesos y la representación gráfica de los indicadores.

Bajo la perspectiva de BPM como un enfoque integral Jeston y Nelis (2006) y Harmon (2007) han introducido una serie de dimensiones para comprender los diferentes niveles del proyecto de BPM (Figura 1).

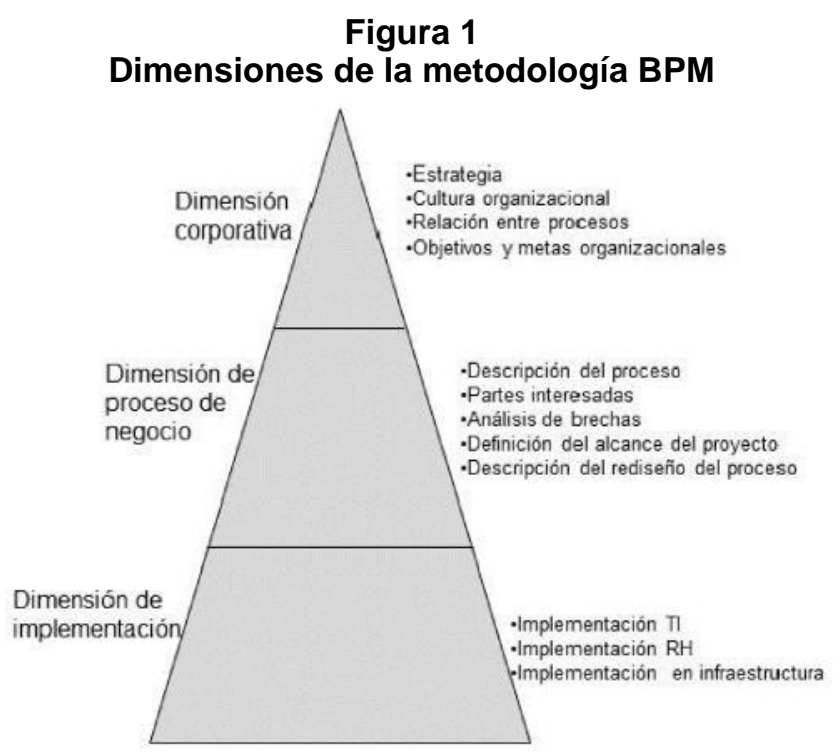

Fuente: Harmon (2007: 354). 
La metodología de BPM cuenta con la dimensión corporativa para documentar la manera en que la compañía planea cumplir con sus objetivos estratégicos. Se consideran características internas como la cadena de valor, donde se analiza su relación con la estructura organizacional y los indicadores de gestión (Yu-Yuan Hung, 2006). Con esta información se busca determinar cómo cada proceso y unidad funcional pueden colaborar a cumplir con la estrategia de la compañía.

En la dimensión de procesos de negocio se modelan los procesos que se verán involucrados en el proyecto de mejora hasta el nivel de descripción necesario, para lo cual se recomienda el uso de la notación BPMN 2.0. Esta notación es un lenguaje gráfico estandarizado de descripción de procesos de negocio, aceptado oficialmente en el año 2006 por la OMG (Object Management Group), organización reconocida mundialmente por estandarizar lenguajes de programación, como el Unified Modelling Language o Lenguaje Unificado de Modelado en español (UML) (Allweyer, 2010).

Como parte de la modelación de los procesos se debe tener en cuenta los insumos y resultados, así como la responsabilidades de las áreas funcionales en el desempeño del proceso y los indicadores de gestión (Kohlbacher y Gruenwald, 2011). Adicionalmente, debe llevarse a cabo un análisis cuantitativo del proceso con el fin de comparar el desempeño con las expectativas de los clientes, ya sean internos o externos. La diferencia entre la situación actual (ASIS), y el deber ser (TO-BE), es el análisis de brechas. En este análisis se empieza a visualizar la magnitud del cambio necesario; y de acuerdo a Jeston y Nelis (2006) deben incluirse los siguientes elementos: descripción del proceso actual, descripción del deber ser, problemática del proceso, brecha de desempeño entre el proceso actual y el deber ser, brecha de capacidades entre el proceso actual y el deber ser.

Con la identificación de las oportunidades de mejora que se pueden lograr con el proyecto, es posible determinar las unidades o áreas de la organización que se verán afectadas, y que por tanto deben participar en el proyecto de mejora. Éstas se relacionan en el diagrama de alcance del proceso (Harmon, 2007).

En la dimensión de implementación se encuentran diferentes aproximaciones de autores que interpretan BPM como una filosofía de mejora de negocios. Se reconoce que se pueden emplear diferentes enfoques de acuerdo con las necesidades de las propuestas de mejora planteadas en el análisis de brechas (Bucher y Winter, 2009; Harmon, 2007; Lee y Dale, 1998; Kohlbacher y Gruenwald, 2011). Mientras que autores que asumen el BPM como un sistema orientado a la tecnología, la implementación se basa en el uso de un sistema BPMS (Jeston y Nelis, 2006; Ko et al., 2009).

En la encuesta llevada a cabo por Ravesteyn y Batenburg (2010) a usuarios de BPM en países bajos, se encuentra que no hay una definición clara de BPMS en la industria. En un intento por definirlo, se valida la definición como una aplicación de software, o suite, que permite el modelamiento, ejecución, monitoreo y representación del proceso de negocio, mediante la integración de funciones de sistemas existentes desarrollado, y orquestados mediante servicios. Esta definición 
concuerda con la definición de BPMS de Harmon (2007), que establece que es un producto de software que combina flujos de trabajo (workflow) y herramientas gerenciales con herramientas de modelación y automatización de procesos soportados en tecnologías de internet.

Dentro de las funciones existentes de los sistemas BPMS se encuentran los protocolos de comunicación entre aplicaciones como los servicios WEB (Tsalgatidou y Pilioura, 2002) y la aplicación del paradigma de $\mathrm{TI}$ de la arquitectura orientada a los servicios (SOA), la cual busca integrar apropiadamente los diferentes módulos tecnológicos con que se soportan los diferentes procesos de negocios, al emplear un bus empresarial que permite su documentación e integración, facilitando la agilidad de cambios tecnológicos al emplear protocolos de servicios WEB (Papazoglou y van den Heuvel, 2007).

\section{Seis Sigma}

La metodología Seis Sigma se dio a conocer por los resultados obtenidos en Motorola al final de la década de los 80 . Mediante la reducción de la variabilidad de los procesos con metas en número de defectos por millón, lograron hacer frente a la competencia japonesa (Linderman et al., 2003), y demostraron retorno sobre la inversión realizada en el proyecto de mejora (Jiju y Banuelas, 2002). La meta medida en partes por millón hace referencia en un proceso con capacidad de proceso en términos de seis desviaciones estándar.

Seis Sigma emplea las técnicas tradicionales de calidad, teniendo en cuenta las condiciones organizacionales, usando un enfoque sistemático para en- contrar las causas de variabilidad del proceso (Schroedor et al., 2008). Jiju y Banuelas (2002) realizaron una encuesta en el Reino Unido sobre el uso de las técnicas y herramientas utilizadas por grandes compañías que implementaron proyectos seis sigma, demostrando en sus resultados que en todos los proyectos se utilizaron diagramas de causa efecto, gráficos de control y análisis de Pareto. Otras herramientas comúnmente usadas fueron: gráficos de tendencia, capacidad de proceso y diseño de experimentos.

El sistema estructurado de Seis Sigma para la mejora de procesos existentes es la metodología Definir, Medir, Analizar, Mejorar, Controlar -DMAIC-: (Sleeper, 2006; Allen, 2006 y Li et al., 2011).

Definir: en esta etapa se conforma el equipo y se realiza la declaración del problema, identificación de las variables críticas de entrada del proceso (KIV) y las variables críticas de salida (KOV).

Medir: en esta etapa se realiza la medición cuantitativa del desempeño y la relación entre KIV y KOV, garantizando un estudio representativo del proceso en términos de número de muestras y tiempo de análisis. En esta etapa se hace uso de las herramientas estadísticas para medir el desempeño actual del proceso.

Analizar: en esta etapa se identifican causas del problema, oportunidades de mejora y variables que deben controlarse para disminuir la variabilidad en los procesos.

Mejorar: se implementan las propuestas de mejora buscando incrementar el desempeño del proceso.

Controlar: se seleccionan e implementar métodos para controlar futuras variaciones de proceso. 
Lo anteriormente expuesto evidencia que la doctrina de Seis Sigma se basa en el mejoramiento de procesos bajo las premisas de que los procesos pueden medirse y analizase, si bien la metodología de BPM se ve también como una metodología para le mejora de procesos, se encuentran otras aplicaciones en la industria dada la amplia definición que posee. En la siguiente sección, se muestra el caso de estudio que ejemplifica su amplio campo de acción y se identifican algunos elementos importantes en su implementación.

\section{BPM y seis sigma en el proceso de compras en una institución universitaria: proyecto de mejora}

En esa sección se analiza el proceso de compras de una universidad privada que cuenta con más de 25.000 estudiantes a nivel de pregrados, especializaciones, maestrías y doctorados. Dentro de la cadena de valor, este proceso, se encuentra como un proceso de soporte y maneja adquisiciones de bienes y servicios con un presupuesto de 10 millones de dólares anuales. El área de suministros es responsable de gestionar las compras de todas las unidades de la universidad que corresponden a los departamentos, facultades, centros de investigación y servicios universitarios.

Inserto en la estrategia definida por la universidad se incluye la importancia de la satisfacción del cliente interno con el fin de retener el talento necesario para la docencia, la investigación y los servicios externos. En el 2010 se concluyó la im- plementación de un sistema integrado de información ERP y a partir de esta fecha todos los procesos de compras en la Universidad se realizan con el soporte de esta herramienta.

Los indicadores de gestión del proceso se basan en el número de solicitudes gestionadas por el área, sin tener en cuenta los tiempos de ciclo ni los responsables, lo cual se identifica como una oportunidad de mejora. El tiempo de ciclo de la compra es uno de los indicadores críticos que impactan en la operación de las unidades.

El proceso de compras se inicia en las unidades, donde se establecen las necesidades que van al área de suministros encargada de gestionar la solicitud y de mantener las relaciones con los proveedores. El proceso de compras actual (AS-IS) consta de cuatro macro actividades que se muestran en la Figura 2. Las unidades de la Universidad generan la solicitud de compra de bienes, el área de suministros se encarga de gestionar la solicitud con el proveedor hasta que el área solicitante recibe el producto y registra la factura del proveedor en el sistema de información.

El detalle del flujo del proceso se muestra en la Figura 3, donde se encuentran: actividades, responsables y decisiones. En el subproceso gestionar solicitud se involucran cinco roles internos: 1) unidad que solicita la compra, 2) analista de compras responsable por la gestión de la compra, 3) responsable de la aprobación (de acuerdo al monto de la compra jefe de suministros, junta de compras o vicerrector) y 3 ) proveedor externo a la organización. 
Business Process Management y Seis Sigma en el análisis de procesos:... Rincon G., Nicolas; Aguirre M., Hugo Santiago y Caballero V., Juan Pablo

Figura 2

Proceso de compras en notación BPMN.

Diagrama de nivel 1

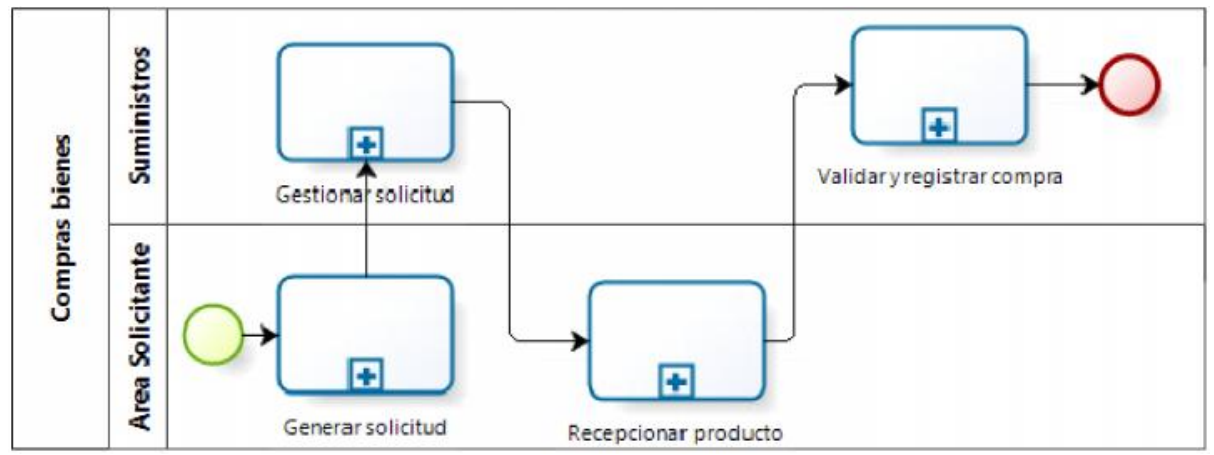

Fuente: Elaboración propia.

Figura 3

Subproceso: gestionar solicitud en notación BPMN

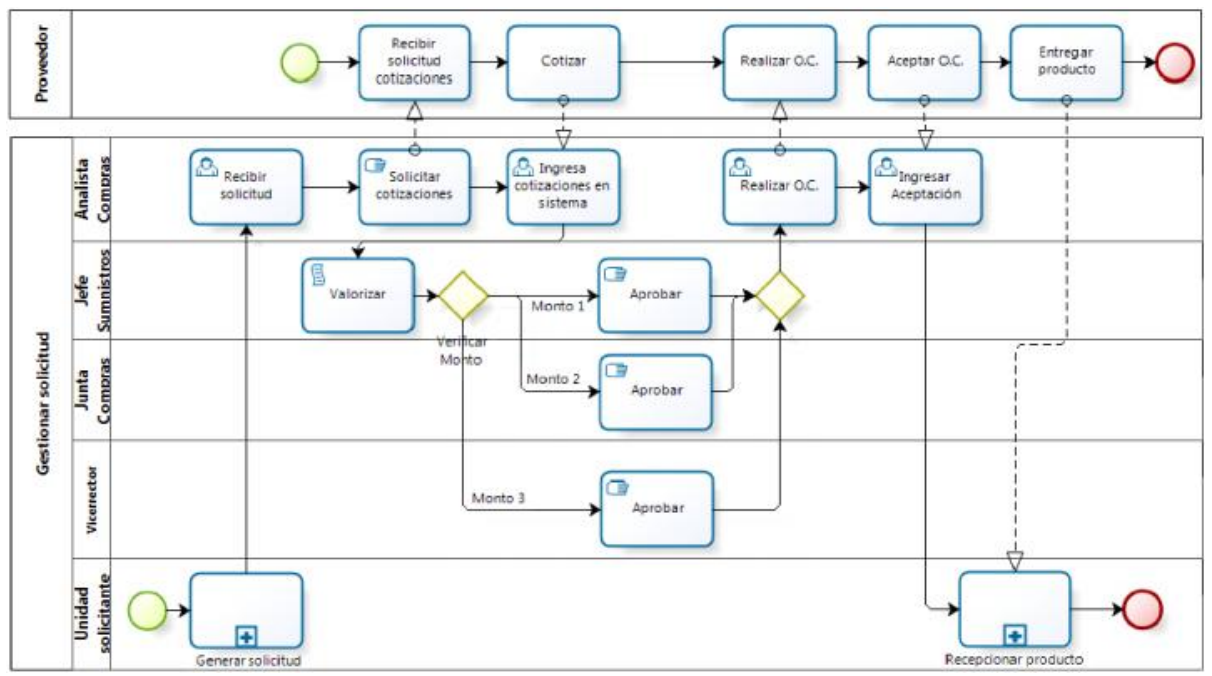

Fuente: Elaboración propia. 
Una vez se establecen los responsables de los procesos y los usuarios del proceso, se identifican las partes interesadas, los cuales harán parte del diagrama de alcance del proceso, donde se identifican las entidades que serán partes activas en la mejora y son la base del análisis del proceso.

Al realizar el análisis cualitativo del proceso bajo la metodología de BPM, se documenta de manera estructurada el conocimiento de los responsables del proceso, el planteamiento de las oportunidades de mejora y entender como el proceso se relaciona con la estrategia de la organización (Harmon, 2007). El análisis se llevó a cabo mediante el análisis de la documentación y reuniones con las partes interesadas lo que permitió identificar una serie de posibles oportunidades de mejora. Estas necesitaron de un estudio cuantitativo para identificar y dimen- sionar la problemática del proceso. Como puntos críticos se encontraron: migración al nuevo sistema de información, falta de indicadores y generación automática de alarmas sobre el estado de las solicitudes (Cuadro 1).

En el diagrama de alcance del proceso (Figura 4) se identifican las oportunidades de mejora relacionados con las diferentes partes interesadas y sus elementos de entrada y salida con el proceso, empleando la herramienta gráfica planteada por Harmon (2007). Los números dentro del diagrama hacen relación a las problemáticas del proceso, el símbolo de atención indica que debe realizarse un estudio de profundización.

En esta etapa del proyecto, se evidencia que no se dispone de indicadores que demuestren el desempeño del proceso y la productividad de los responsables del mismo.

\section{Cuadro 1 \\ Oportunidades de mejora del proceso de compras}

\begin{tabular}{clc}
\hline Identificación & \multicolumn{1}{c}{ Descripción oportunidad de mejora } & Estado \\
\hline 1 & $\begin{array}{l}\text { Dado la implantación del nuevo sistema de información, no } \\
\text { se han registrado todas las compras adecuadamente en el } \\
\text { ERP. Se observan actividades no automatizadas dentro del } \\
\text { proceso de compras (solicitud de cotizaciones: tarea manual } \\
\text { ejecutada con la ayuda de una hoja de cálculo). }\end{array}$ & Profundización \\
& $\begin{array}{l}\text { El seguimiento a las compras se realiza de forma manual, } \\
\text { apoyada en una hoja de cálculo. No existe control del } \\
\text { proceso ni alarmas automáticas ante retrasos. }\end{array}$ & Profundización \\
& $\begin{array}{l}\text { No se cuenta con un sistema de indicadores que midan la } \\
\text { gestión de los diferentes responsables de la ejecución del } \\
\text { proceso en términos de tiempo. Se desconoce el } \\
\text { comportamiento del proceso. }\end{array}$ & Identificada \\
& $\begin{array}{l}\text { No se cuenta con una herramienta de generación automática } \\
\text { de reportes con el fin de disponer de información en tiempo } \\
\text { real para evitar retrasos en las compras. }\end{array}$ & Profundización \\
& & \\
\hline
\end{tabular}

Fuente: Elaboración propia. 


\section{Figura 4 \\ Herramienta de BPM. \\ Diagrama de alcance: diagnóstico proceso compras}

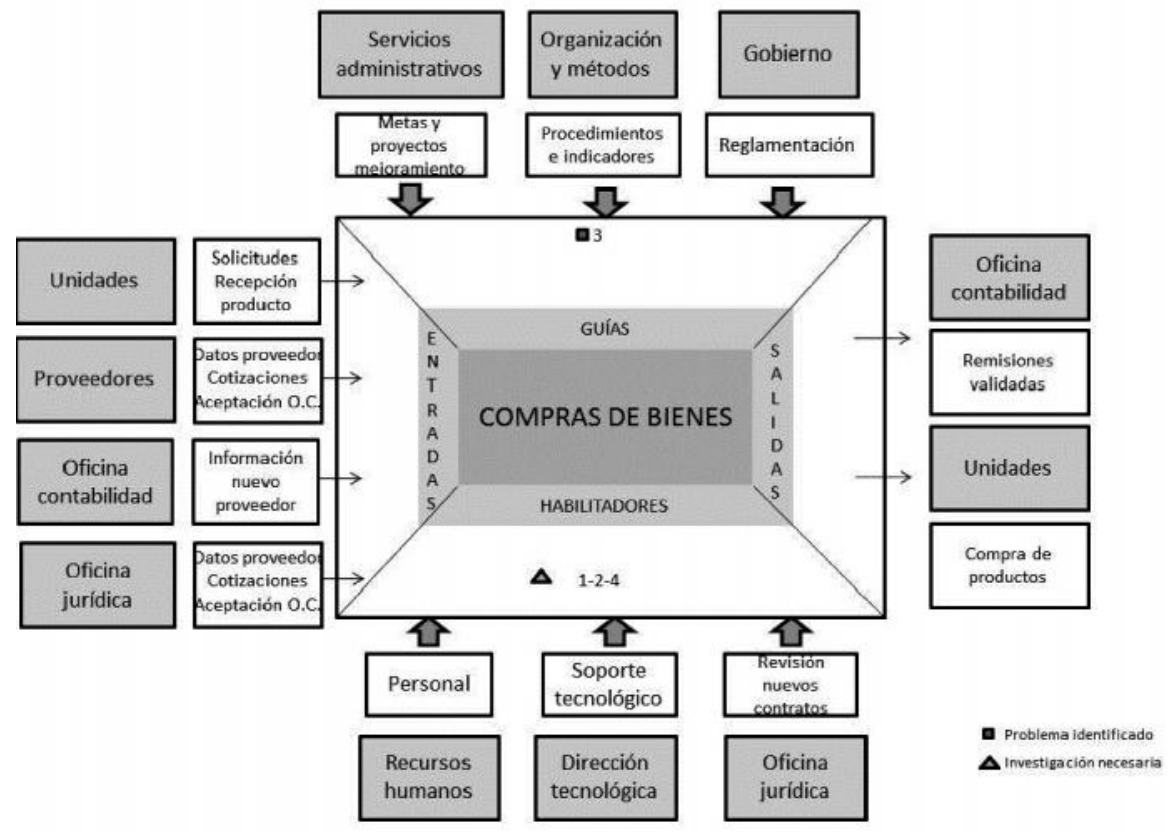

Fuente: Elaboración propia.

Para realizar el análisis cuantitativo del proceso del proceso de compras, Seis Sigma cuenta con una serie de herramientas. Does et al. (2002) considera el uso de la metodología en ocho proyectos de Seis Sigma llevados a cabo en empresas de servicio en Holanda. En estos proyectos el estudio del desempeño del proceso se llevó a cabo en organizaciones de servicio, en ellas fue necesario hacer énfasis en el análisis de media y desviación. El análisis estadístico del proceso (SPC) y el uso de gráficos de control en procesos de servicio (Furterer y Elshennaway, 2005), permite monitorear, con- trolar, analizar y mejorar los procesos usando métodos estadísticos (Mason y Jiju, 2000).

De acuerdo a Herbert et al. (2003), existe una diferencia entre la utilización de estas técnicas en manufactura y servicios. Mientras que en la manufactura se enfoca en identificar y mejorar las condiciones que generan productos fuera de tolerancia, en los servicios se emplea para evaluar el desempeño del proceso, dar guía para la mejora y soportar la toma de decisiones de la gerencia.

Con el fin de analizar el comportamiento del proceso se diseñaron una se- 
rie de indicadores basados en los tiempos de las actividades de la tarea gestionar solicitud (Figura 5). Para esto fue necesario analizar en un diagrama el detalle de la tarea gestionar solicitud y el soporte informático de cada una de las actividades, con el fin de extraer los datos de los eventos almacenados en el ERP mediante una consulta específicamente diseñada con este fin.

El tiempo de proceso: orden de compra (O.C.) mide el tiempo que toma procesar una solicitud hasta que se genera la orden de compra, mientras que el tiempo de compra tiene en cuenta el tiempo de entrega del proveedor. Para calcular estos tiempos se incluyó todas las solicitudes procesadas en el periodo de tiempo analizado sin tener que recurrir al muestreo gracias a la generación de la consulta en el sistema ERP.

El análisis de los indicadores se realizó para el segundo semestre del primer año buscando información del proceso una vez se hubiese estabilizado después de la implementación del ERP. Se observa que dentro del proceso se encuentran 16 familias de productos, de los cuales se decidió analizar 4 familias, que fueron seleccionadas de acuerdo a los criterios de mayor número de compras y criticidad. El análisis de los tiempos del proceso se presenta en la Tabla 1.

Al analizar los tiempos promedio de las etapas de la tarea gestionar solicitud en las diferentes familias se encuentran diferencias. El tiempo total de proceso de O.C. de la familia 2 es casi el doble de la familia 3. Adicionalmente, se identifica una gran variabilidad dentro de los tiempos totales de proceso de O.C. entre las familias 1,3 y 4 , donde la desviación estándar es mayor que el tiempo promedio.

En el análisis de tiempos se identifica que el mayor tiempo del proceso gestionar solicitud corresponde a la suma de los tiempos Cotizar y Envío O.C. a proveedor, lo cual es responsabilidad de los analistas de compra. Debido a lo anterior se decidió analizar el tiempo de proceso de O.C. con el uso de los gráficos de control para observar el comportamiento del proceso a través del tiempo.

De acuerdo a Mason y Jiju (2000) los gráficos de control típicos para variables de tiempo de proceso en los servicios son $\bar{X} R$ y $\bar{X} S$, en los cuales se grafica el promedio de cada muestra en la gráfica $\bar{X}$ y su varia-

\section{Figura 5}

Tarea gestionar solicitud: Tiempo de las actividades

\begin{tabular}{|c|c|c|c|c|c|}
\hline $\begin{array}{c}\text { Solicitud de compra } \\
\text { aprobada por } \\
\text { unidad }\end{array}$ & Cotizar & $\begin{array}{c}\text { Aprobar } \\
\text { compra }\end{array}$ & $\begin{array}{c}\text { Enviar O.C. } \\
\text { a proveedor }\end{array}$ & $\begin{array}{c}\text { Recibir } \\
\text { mercancía }\end{array}$ & $\begin{array}{c}\text { Generar } \\
\text { registro }\end{array}$ \\
\hline
\end{tabular}

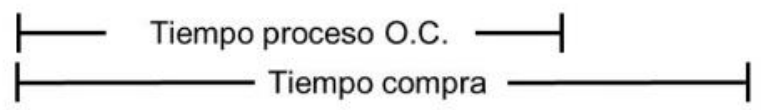

Fuente: Elaboración propia. 


\section{Tabla 1 \\ Análisis de tiempo promedio de la tarea gestionar solicitud (expresado en días)}

\begin{tabular}{lcccccc}
\hline & & $\begin{array}{c}\text { Tiempo } \\
\text { cotizar }\end{array}$ & $\begin{array}{c}\text { Tiempo } \\
\text { aprobar } \\
\text { compra }\end{array}$ & $\begin{array}{c}\text { Tiempo } \\
\text { enviar } \\
\text { O.C. } \\
\text { proveedor }\end{array}$ & $\begin{array}{c}\text { Tiempo } \\
\text { Total } \\
\text { proceso } \\
\text { O.C. }\end{array}$ & $\begin{array}{c}\text { Tiempo en } \\
\text { recibir } \\
\text { mercancía }\end{array}$ \\
\hline Familia 1 & Promedio & 8,18 & 3,1 & 2,0 & 13,3 & 10,9 \\
Familia 2 & Promedio & 13,5 & 4,1 & 7,1 & 24,8 & 17,4 \\
& $\mathrm{~S}$ & 11,4 & 4,8 & 11,1 & 21,4 & 20,3 \\
Familia 3 & Promedio & 4,5 & 2,6 & 0,8 & 7,9 & 14,1 \\
& $\mathrm{~S}$ & 5,1 & 3,4 & 0,8 & 7,0 & 17,4 \\
Familia 4 & Promedio & 6,5 & 3,3 & 2,2 & 12,0 & 28,8 \\
& $\mathrm{~S}$ & 10,3 & 6,2 & 4,7 & 13,4 & 28,8 \\
\hline
\end{tabular}

Fuente: Elaboración propia.

ción en la gráfica $\mathrm{R}$ ó S. Los límites de control se calculan a partir de constantes que se usan para estimar los valores de la desviación de la población a partir de las muestras seleccionadas y sirven de referencia para identificar causas especiales de variación (Evans y Lindsay, 2000). Se seleccionó el gráfico $\bar{X}$-S con tamaño de muestra 5 con el fin de trabajar bajo el supuesto de normalidad (Allen, 2006). Las fórmulas para el cálculo de los límites de control se muestran en la Figura 6.

En la Figura 7 se encuentra el gráfico $\bar{X}$ y en la Figura 8 se muestra el gráfico $S$ para la familia 1 , la cual es considerada de fácil consecución. Se identifica que el proceso está fuera de control, al revisar los puntos fuera de los límites de control se encuentran procesos con gran variación de tiempo y una alta desviación. Se plantean como variables críticas de entrada al proceso (KIV) la familia del producto a comprar y el comprador.
Con el fin de probar la significancia estadística del efecto de la familia y el comprador en el tiempo de proceso de O.C. se efectuaron una serie de análisis estadísticos. El análisis de varianza ó ANOVA es una herramienta para probar la hipótesis de que la media de tiempo de proceso varía de acuerdo con las variables criticas de entrada, pero al analizar los datos se violaban los supuestos del ANOVA sobre normalidad de la población e igualdad de las desviaciones de la población (Allen, 2006). Razón por la cual se empleó la prueba de Tamhane, donde se encontró significancia estadística al nivel de 5 por ciento para la diferencia de medias entre compradores y entre familias.

Dado que diferentes analistas compran elementos de una misma familia de productos se analiza el desempeño de los 2 analistas con mayor volumen de compras en la familia de productos 1 . El analista 1 tiene un promedio de 19.13 
Figura 6

Fórmulas para gráfico de control $\bar{X}$ y $S$

$$
\begin{gathered}
\overline{\bar{X}}=\frac{\sum_{i=1}^{k} X_{i}}{k} \quad S_{j}=\sqrt{\frac{\sum_{i=1}^{5}\left(X_{i}-\bar{X}_{j}\right)^{2}}{4}} \\
\bar{S}=\frac{\sum_{j=1}^{1} S_{j}}{k}
\end{gathered}
$$

Donde $k$ es el número de registros validos para la familia,

$\mathrm{S}_{\mathrm{j}}$ es la desviación de la muestraj-esima y J es el número de muestras.

Gráfico $\bar{X}$

$$
\begin{aligned}
& \text { Limite superior }=\overline{\bar{X}}+A_{3} \bar{S} \\
& \text { Limite inferior }=\overline{\bar{X}}-A_{3} \bar{S}
\end{aligned}
$$

Donde $A_{3}, B_{3}$ y $B_{4}$ son constantes de factores para gráficos de control.

Fuente: Evans y Lindsay, 2000.

\section{Figura 7 \\ Gráfico de control $\bar{X}$ para la familia 1}
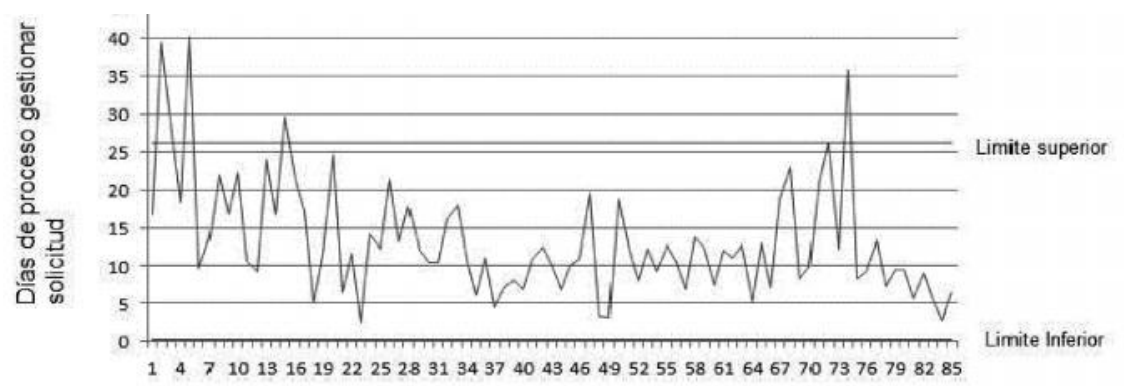

Consecutivo de muestras con tamaño 5 para la familia 1

Fuente: Elaboración propia.

días en tiempo de proceso O.C. y una desviación de 13.04 mientras que el analista 2 tiene un promedio de 11.19 días y una desviación de 11.16. El analista 2 realiza el proceso en menor tiempo y con menor variación, mostrando un compor- tamiento similar en las diferentes familias.

Debido a que el área de suministros no cuenta con indicadores basados en el tiempo de respuesta, los analistas de compras no tienen una meta del tiem- 


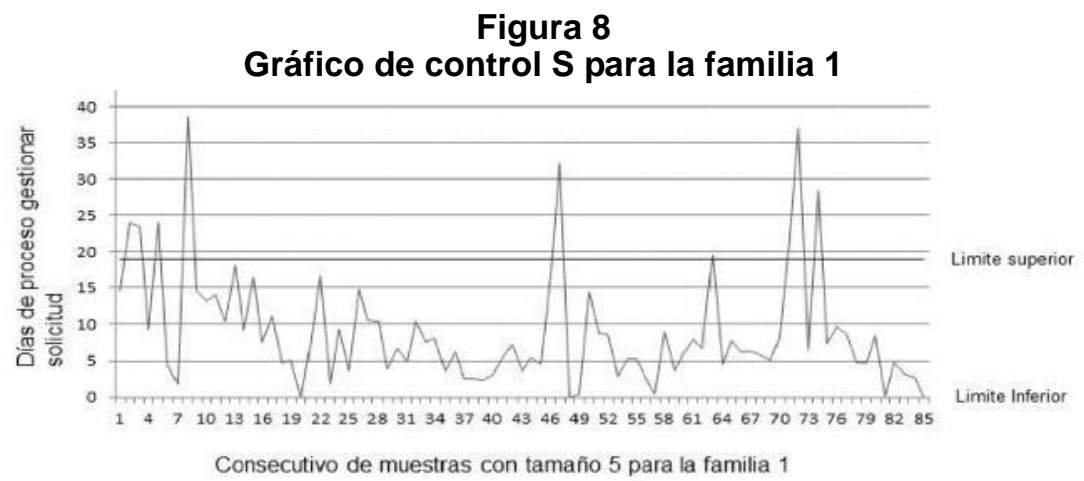

Fuente: Elaboración propia.

po esperado para procesar las solicitudes de compra. Los indicadores deben permitir la toma de decisiones por parte de los responsables de la operación y los líderes del proceso, por lo que se propone la generación de un sistema de indicadores con unas metas claramente establecidas para los analistas de compras.

Con el fin de tener un sistema de indicadores de gestión del proceso es necesario contar con una herramienta que permita realizar la medición automática de los tiempos de proceso. En el análisis del soporte tecnológico se encuentra que no existe una generación automática de alarmas de procesos que presentan retrasos. Estas oportunidades de mejora podrían implementarse con una herramienta automatizada que obtenga los datos de la base de datos del ERP y genere los indicadores y alarmas, lo que podría configurarse de acuerdo con el análisis cualitativo obtenido mediante el análisis estadístico y las metas establecidas por el área.

Los eventos que deben hacer parte de la herramienta de indicadores se obtienen gracias al análisis de datos que se realizó para diagnosticar el proceso de compras, para ello se puede generar un desarrollo en el ERP o generar una aplicación WEB que se integre con la base de datos existente (Vega Mejia et al., 2011).

Para finalizar esta etapa, en el análisis de brechas de la metodología BPM (Figura 9) se encuentra el resumen del diagnóstico, las oportunidades de mejora, los valores actuales del comportamiento del proceso, valores esperados con el proyecto y los cambios en el proceso requerido para lograrlo. El diagrama de brechas es una herramienta gráfica que resume los objetivos del proyecto de forma cuantitativa y cualitativa (Harmon, 2007).

El análisis de brechas tiene dos elementos: desempeño y capacidades. En la brecha de desempeño se establece la diferencia entre los indicadores actuales del proceso y el nivel deseado en estos indicadores (Harmon, 2007). Las metas propuestas del proyecto se basan en reducir la variabilidad del proceso, actualmente el $51 \%$ de las solicitudes de compra de la familia 1 toma menos de 20 días en generar la O.C. En la brecha de capacidades se mencionan las acciones que deben implementarse para alcanzar el 


\section{Figura 9}

Análisis de brechas

Proyecto mejora del proceso de compra de bienes en universidad

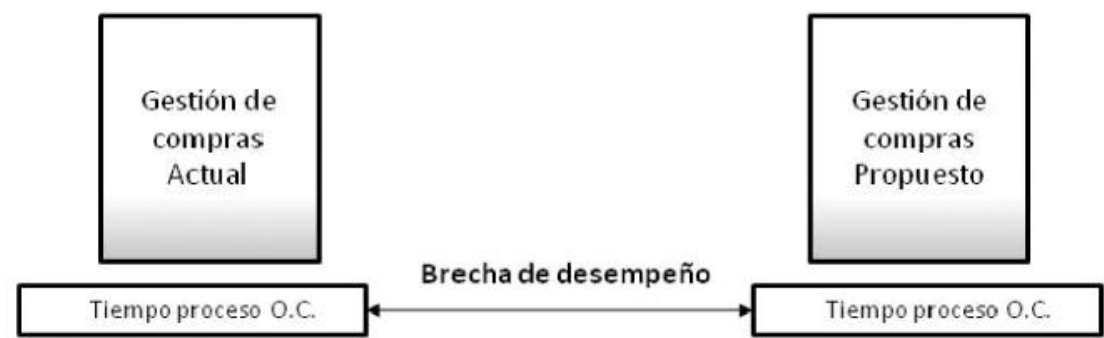

$=0-3=4-10=10-20=20-40=40-65=65-90$
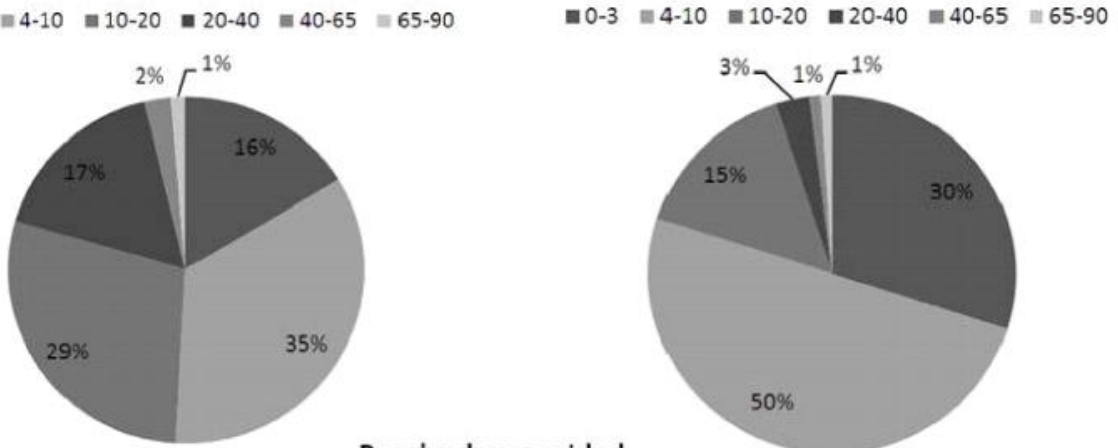

Brecha de capacidad

Proceso compras Actual

Proceso compras Propuesto

El proceso no cuentacon indicadores que midan la gestión de los ejecutores.

No se cuenta con una herramienta que permita generar alertas sobre retrasos de las solicitudes, ni que genere indicadores en tiempo real para agilizar el proceso.
Contar con indicadores que midan la gestión de los ejecutores.

Contar con una herramienta que permita generar alertas sobre retrasos de las solicitudes, y que genere indicadores en tiempo real para poder tomar decisiones que agilicen el proceso de gestión solicitud.

Fuente: Elaboración propia. 
desempeño deseado del proceso (Harmon, 2007). Se espera que mediante un sistema de gestión basado en indicadores y metas, el cual sea soportado por una herramienta automática de generación de indicadores, se logre que el $80 \%$ en las compras de la familia 1 se gestione en menos de 20 días y se reduzca el número de solicitudes con retrasos superiores a 20 días.

El caso de estudio permitió verificar que las herramientas de BPM y Seis Sigma se complementan. El enfoque cualitativo de BPM permite alinear el pro- yecto de mejora con la estrategia de la organización, documentar el proceso, el conocimiento de las partes interesadas y diseñar los indicadores relevantes para identificar las oportunidades de mejora. El análisis del soporte tecnológico permite obtener la información para el análisis cuantitativo de los indicadores y plantear soluciones basado en la automatización en la consecución de los indicadores de gestión del proceso. Los elementos empleados en el diagnóstico de cada una de las metodologías se muestran en el cuadro 2.

\section{Cuadro 2 \\ Integración de BPM y Seis Sigma para el diagnóstico y análisis del proceso}

\begin{tabular}{|c|c|c|}
\hline Aspecto & Elementos de BPM & Elementos de Seis Sigma \\
\hline $\begin{array}{l}\text { Descripción del } \\
\text { proceso actual }\end{array}$ & $\begin{array}{l}\text { BPMN } \\
\text { Diagrama de partes interesadas }\end{array}$ & $\begin{array}{l}\text { Definir: Conformación del equipo y } \\
\text { declaración del problema. }\end{array}$ \\
\hline $\begin{array}{l}\text { Descripción del } \\
\text { deber ser }\end{array}$ & $\begin{array}{l}\text { Se emplea la dimensión corporativa } \\
\text { para alinear el proyecto de mejora } \\
\text { con la estrategia de la compañía. }\end{array}$ & \\
\hline $\begin{array}{l}\text { Problemática del } \\
\text { proceso }\end{array}$ & $\begin{array}{l}\text { Identificación de oportunidades de } \\
\text { mejora a partir del conocimiento de } \\
\text { las partes interesadas empleando } \\
\text { la herramienta Diagrama de } \\
\text { alcance. } \\
\text { Diseño de indicadores a partir de } \\
\text { la descripción del proceso y su } \\
\text { análisis. } \\
\text { Análisis del soporte informático } \\
\text { para obtener datos. }\end{array}$ & $\begin{array}{l}\text { Definir: Identificación de KIV y KOV. } \\
\text { Medir: Evaluación cuantitativa del } \\
\text { desempeño; análisis de la relación } \\
\text { entre KIV Y KOV mediante } \\
\text { herramientas estadísticas como } \\
\text { gráficos de control y análisis de } \\
\text { variabilidad de medias. }\end{array}$ \\
\hline $\begin{array}{l}\text { Brecha de } \\
\text { desempeño }\end{array}$ & & $\begin{array}{l}\text { Análisis: Análisis del impacto sobre el } \\
\text { desempeño esperado del proceso al } \\
\text { controlar las KIV. }\end{array}$ \\
\hline $\begin{array}{l}\text { Brecha de } \\
\text { capacidades }\end{array}$ & Automatización de indicadores. & $\begin{array}{l}\text { Elementos para parametrizar la } \\
\text { automatización de indicadores }\end{array}$ \\
\hline
\end{tabular}

Fuente: Elaboración propia. 
Por otro lado Seis Sigma ofrece un enfoque cuantitativo con el fin de dar un soporte estadístico a la identificación de oportunidades de mejora (Harmon, 2007; Neubauer 2009; Johansen et al. (2010). El uso del enfoque cuantitativo en procesos de servicios presenta retos al ser de mayor complejidad la definición de métricas que en los procesos de manufactura (Does et al., 2002). El análisis cualitativo de BPM provee herramientas para identificar las métricas necesarias mediante el análisis del flujo del proceso y análisis del sistema de información.

Las herramientas cuantitativas de Seis Sigma pueden aprovecharse ampliamente ya que los sistemas ERP que actualmente soportan los procesos en las organizaciones permiten obtener una gran cantidad de datos y registros, así como analizar largos periodos de tiempo. De esta forma se logra un soporte estadístico para la cuantificación de las oportunidades de mejora y el impacto de controlar las variables críticas de entrada sobre el desempeño del proceso.

\section{Conclusiones}

BPM y Seis Sigma son enfoques de mejora complementarios. BPM permite a una organización alinear los procesos con la estrategia del negocio y tiene una metodología lógica de mejora de procesos basado en el planteamiento del problema, análisis, rediseño e implementación. Seis Sigma complementa el enfoque de mejora de BPM con herramientas estadísticas para la etapa de análisis de procesos.
El análisis de brechas en el planteamiento de un proyecto de mejora permite determinar la diferencia entre los indicadores de desempeño alcanzados por el proceso y el nivel deseado en estos indicadores. Esto permite alinear los objetivos de mejora de los procesos con los objetivos estratégicos de la organización y las necesidades de los clientes del proceso.

El control estadístico de procesos de Seis Sigma es una herramienta de gran utilidad para obtener el diagnóstico del desempeño del proceso y determinar la causa raíz de los problemas del proceso. Estas técnicas cuantitativas se deben complementar con herramientas cualitativas como son el análisis del flujo del proceso, la documentación de forma estructurada del proyecto de mejoramiento y el análisis del soporte tecnológico del proceso, para obtener un análisis que permita identificar de forma objetiva las oportunidades de mejora.

En el caso de aplicación se pudo comprobar que los enfoques de mejora de BPM y Seis Sigma se complementan para lograr un diagnóstico y análisis integral de un proceso de negocio. Si bien existen puntos en común en los pasos de mejora de ambas metodologías, las herramientas son complementarias y se pueden usar para obtener un análisis cuantitativo y cualitativo para la mejora y rediseño de procesos.

Como trabajo futuro se plantea el estudio de la integración de las herramientas de BPM y Seis Sigma en otro tipo de procesos y organizaciones, como pueden ser procesos logísticos o de manufactura para verificar que la propuesta de este artículo es válida para otros casos de negocio. 


\section{Referencias Bibliográficas}

Aboelmaged, Mohamed G. (2010). Six Sigma quality: a structured research, International Journal of Quality \& Reliability Management, Volumen 27(3), pp. 268-17

Aguirre, Santiago; Parra, Carlos y Alvarado, Jorge (2013). Combination of Process Mining and Simulation techniques for business process redesign: a methodological approach. Lecture Notes In Business Information Processing. Volumen 162. pp.24-43.

Allweyer, Thomas (2010). BPMN 2.0: Introduction to the Standard for Business Process Modeling, Editorial Herstellung und Verlag, $2^{\text {nda }}$ edición, pp. 10

Bucher, Tobias y Winter, Robert (2009). Project types of business process management: Towards a scenario structure to enable situational method engineering for business process management, Business Process Management Journal, Volumen (4), pp 548568.

Climent, Cristina; Mula, Josefa y Hernández, Jorge E. (2009). Improving the business processes of a bank, Business Process Management Journal, Volumen 15(2), pp 201-224.

Does, Ronald; van den Heuvel, Edwin; de Mast, Jeroen y Bisgaard Soren. (2002) Comparing nonmanufacturing with Traditional Applications of Six Sigma. Quality Engineering. Volumen 15(1), pp 177-182.

Dubois, Anna y Araujo, Luis (2007). Case research in purchasing and supply management: opportunities and challenges, Journal of Purchasing \& Supply Management, Volumen 13, pp 170-181.

Evans, James R. y Lindsay, William M. (2000). Administración y control de la cali- dad, editorial Thompson, 8va edición, pp. 652-669.

Furterer, Sandra y Elshennawy, Ahmad, K. (2005). Implementation of TQM and Lean Six Sigma Tools in Local Government: a Framework and a Case Study. Total Quality Management. Volumen 16(10). pp 1179-1191.

Han, Kwan H.; Kang, Jin G. y Song, Minseok (2009). Two-stage process analysis using the process-based performance measurement framework and business process simulation, Expert Systems with Applications, Volumen 36, pp 7080-7086.

Harmon, Paul (2007). Business Process Change: A Guide for Business Managers and BPM and Six Sigma Professionals, $2^{\text {da }}$ edición, Editorial Morgan Kaufmann Publishers, pp. 447-471.

Herbert, David; Curry, Adrienne y Angel Leon (2003). Use of quality tools and techniques in services, The Service Industries Journal, Volumen 23(4), pp 61-80.

Hesson, Mihyar; Al-Ameed, Hayder y Samaka, Muhammad (2007). Business process reengineering in UAE public sector: a town planning case study. Business Process Management Journal, Volumen 13 (3), pp 348-378.

Jeston, John y Nelis, Johan (2006). Business Process Management: Practical Guidelines to successful implementations, Oxford, UK: Butterworth-Heinemann Elsevier.

Jiang, Wei; AU, Tom y TSUI, Knok-Leung (2007). Statistical process control approach to business activity monitoring. IIE Transactions, Volumen 39, pp 235-249.

Jiju, Antony y Banuelas, Ricardo (2002). Key ingredients for the effective implementation of Six Sigma, Measuring Business Excellence, Volumen 6 (4), pp 20-27. 
Johannsen, Florian; Leist, Sussane y Zellner, Gregor (2010). Six sigma as a business process management method in services: analysis of key application problems, Information Systems and E-Business Management Journal, Volumen 9 (3), pp 307-332.

Ko, Ryan K.L; Lee, Stephen y Lee, Eng Wah (2009). Business process management. (BPM) standards: a survey, Business Process Management Journal, Vol.15, No.5, Emerald group publishing Limited, pp. 744-791.

Küng, Peter y Hagen, Claus (2007). The fruits of Business Process Management: an experience report from a Swiss bank, Business Process Management Journal, Volumen 13(4), pp 477-487.

Kohlbacher, Markus y Gruenwald, Stefan (2011). Process Orientation: conceptualization and measurement, Business Process Management Journal, Volumen 17(2), pp 267-283.

Lederer, Antonucci Y. y Goeke, Richard J. (2011). Identification of appropriate responsibilities and positions for business process management success: Seeking a valid and reliable framework, Business Process Management Journal, Volumen 17 (1), pp 127-146.

Li, Shing-Han; Wu, Chi-Chuan; Yen, David C.; y Lee, Ming-Chih (2011). Improving the efficiency of IT help-desk service by Six Sigma management methodology (DMAIC) - a case study of C company. Production planning \& Control, volumen 22 (7), pp 612-627.

Lee, Roy G. y Barrie G. Dale. (1998). Business Process Management: a review and evaluation. Business Process Management Journal, Volumen 4 (3), pp 214-225

Linderman, Kevin; Schroeder Roger G; Zaheer, Srilata y Choo, Adrian S. (2003). Six sigma: a goal-theoretic perspective, Journal of Operations Management, Volumen 21 (2), pp 193-203.

Mason, Ben y Jiju, Antony (2000). Statistical process control: an essential ingredient for improving service and manufacturing quality, Managing Service Quality, Volumen 10(4), pp 233-238.

Mooney, Laura, Corporate Senior Director, and Product Marketing. " 5 steps to choosing the right BPM suite." 5 steps to choosing the right BPM suite (2006). Disponible en: [http://www.ascot-solutions.co.uk/PDFs/ArticlesWhitePapers/5_Steps_to_Choosing_ the_Right_BPM_Suite.pdf], [Consulta: 27 Marzo, 2014]

Neubauer, Thomas (2009). An empirical study about the status of business process management, Business Process Management Journal, Volumen 15 (2), pp 166-183.

Papazoglou, Mike y van den Heuvel, WillemJan (2007). Service oriented architectures: Approaches, technologies and research issues, Very Large Database Journal, Volumen 16 (3), pp 389415.

Process Excellence Network (2013). 2013 State of the Industry: Process Excellence. Disponible en: [http://www.processexcellencenetwork.com/lean/hite-papers/infographic-process-excellence-state-of-the-indust/], [Consulta: 30 Septiembre, 2013]

Ravesteyn, Pascal y Batenburg, Ronald (2010). Surveying the critical success factors of BPM-systems implementation. Business Process Management Journal, Volumen 16 (3), pp 492-507.

Rebuge, Alvaro y Ferreira, Diogo R. (2012). Business process analysis in healthcare environments: A methodology base on process mining, Information Systems, Volumen 37, pp 99-116. 
Seetharaju, Ravi y Marjanovic, Olivera (2009). Role of process knowledge in business process improvement methodology: a case study, Journal of Business Process Management, Volumen 15 (6), pp 920-936.

Sleeper, Andrew D. (2006). Design for six sigma statistics, McGraw Hill, First edition, pp. 5.

Stanley, Linda L. y Wisner, Joel D. (2002). The determinants of service quality: issues for purchasing, European Journal of Purchasing \& Supply Management, Volumen 8 (2), pp 97-109.

Tiwari, Ashutosh; Turner, Chris. J. y Majeed, Basim M. (2008). A review of business process mining: state-of-the-art and future trends. Business Process Management Journal, Volumen 14 (1), pp 5-22.

Tsalgatidou, Aphrodite y Pilioura, Thomi (2002). An Overview of Standards and Related Technology in web services, International Journal of Distributed and Parallel Databases, Volumen 12 (2), pp 135-162.

Van der Aalst, Wil. M. P; Weske, Mathias y Wirtz, Guido (2003a). Advanced topics in workflow management: issues, requirements, and solutions. Journal of Integrated design \& process science, Volumen 7 (3), 49-77.

Van der Aalst, Wil. M. P.; Hofstade Arthur. H. M. y Weske, Mathias (2003b). Business process management: A survey. Proceedings of the 1st International Conference on Business Process Management, Volumen 2678 of LNCS.

Vega Mejía, Carlos. A.; Castellanos Arias, Johana S.; Aguirre Mayorga, Hugo S.; Rincón, Nicolas y Hernández Martinez, Paola (2011). ERP and BPMS integration at a manufacturing simulation. Robotics Symposium, 2011 IEEE IX Latin American and IEEE Colombian Conference on Automatic Control and Industry Applications (LARC).

Yin, Robert, K. (2003). Case study research: Design and methods. Third edition. Sage Publications.

Yu-Yuan Hung, Richard (2006). Business process management competitive advantage: a review and empirical study, Total Quality Management Business Excellence, Volumen 17 (1), pp 21-40. 